\title{
Developing Multi-Directional Broadcasting for Both Highway and Urban Vehicular Sensor Networks
}

\author{
Minwoo Ryu ${ }^{1}$, Si-Ho Cha ${ }^{2 *}$ \\ ${ }^{1}$ Korea Telecom R\&D Center, KT, Seoul, 06763, South Korea, \\ ${ }^{2}$ Dept. of Multimedia Science, Chungwoon University, 113, Seokgol-ro, Michuhol-gu, \\ Incheon 22100, South Korea (Corresponding Author) \\ 2 shcha@chungwoon.ac.kr
}

\begin{abstract}
Vehicular Sensor Network (VSN) has to ensure the prompt dissemination of critical sensing data to all vehicles within Region of Interest (ROI) to avoid various road dangers. In order for VSN to provide safe road services on both highways and urban roads, it must have broadcasting protocols that can efficiently deliver hazardous messages to all nearby vehicles. This paper proposes a mobility prediction based multi-directional broadcasting (MPMB) protocol for both highway and urban VSNs. The MPMB protocol consists of (i) mobility prediction phase and (ii) broadcasting phase. In the mobility prediction phase, each vehicle predicts the Link Available Time (LAT) of its all neighbor vehicles through periodical beaconing. In the broadcasting phase, each broadcasting vehicle adaptively selects a vehicle with the largest LAT value predicted in the previous mobility prediction phase as a rebroadcasting vehicle each possible directional sector.
\end{abstract}

Keywords: Broadcasting, Vehicular Sensor Networks, Mobility Prediction, Link Available Time, Region of Interest

\section{Introduction}

Vehicular Sensor Networks (VSNs) have been installing for detecting roads and vehicles phenomena by using sensors and transmitting sensor data to relevant vehicles or infrastructures. VSNs are a subset of Vehicular Ad-hoc Networks (VANETs) placed either in vehicles or alongside roads creating an end-to-end reliable network for disseminating sensor data gathered from a vehicular environment [1]. In Intelligent Transportation System (ITS)[2][3], traffic and weather information, road hazard and obstacle information, and traffic accidents are usually disseminated by broadcasting protocol. Therefore, the multiple critical detection data collected by different types of sensors should be disseminated to all surrounding vehicles to prevent secondary accidents. Broadcast is considered to be the most suitable communication mechanism for propagating safety messages in VSNs. Broadcasting is the process of message dissemination by the source vehicle to all other neighboring vehicles on the VSNs. Emergency messages shall be broadcast to all vehicles in the Regions of Interest (ROI) [4] where is an area exposed to potential hazards in the event of a road accident. For secure VSNs, emergency messages should be propagated all neighbor vehicles without a time delay to avoid potential accidents [5][6][7]. In this paper, we propose a mobility-predicting-based Multi-way Broadcast

Article history:

Received (May 13, 2019), Review Result (June 21, 2019), Accepted (August 19, 2019) 
(MPMB) protocol for VSNs that can support both highway and urban scenarios. The MPMB provides out multi-directional broadcasting for both highway and urban scenarios of VSNs. The MPMB adopts adaptive directional sectors, mobility predictions and Store-Carry-Forward (SCF) [8] methods to select the next broadcast (re-broadcasting) vehicle for each possible directional sector. The MPMB protocol consists of two stages: the forecasting stage for mobility and the broadcasting stage. In the forecasting stage of mobility, each vehicle predicts the LAT of all adjacent vehicles through regular beacons. In the broadcasting phase, only one vehicle with the greatest LAT value predicted in the previous stage is selected as the vehicle on which each broadcast vehicle re-runs each directional segment. The broadcast stage then broadcasts a message containing the re-run vehicle ID for each possible directional sector to all adjacent vehicles. The MPMB protocol can reduce unnecessary delays caused by media contention between adjacent vehicles, as the vehicle that is required to re-run the message is determined by the previous hop. MPMB protocols can also improve reliability both on highways and in urban areas by adopting adaptive direction sectors, mobility predictions and SCF methods.

\section{Related Work}

At Store-Carry-Forward (SCF), Ohta et. al proposed a new reliable data delivery method to address redundant data packets. They combined vehicle information with a cognitive path consisting of direction and location information for each vehicle node derived from its Global Positioning System (GPS). Furthermore, they defined two conditions based on vehicle information to select the next node for data packet delivery. This reduces redundant data packets when the vehicle node forwards the data packets to an adjacent node. Korkmaz et. al have proposed an efficient IEEE 802.11-based urban multi-85 broadcasting protocol (UMB) to address the issue of multi-hop broadcasting in the vehicle ad hoc network (VANET) [9]. In particular, they are trying to solve broadcasting storms, hidden nodes, and reliability. In the intersection broadcast method, they also choose the best candidate to be the next node for forwarding data packets to the target using repeaters located at the intersection. Tonguz et. al have proposed a Distributed Vehicle Broadcast Protocol (DV-CAST) for VANET [10]. In their research work, they designed a distributed broadcasting protocol to mitigate broadcast storms and maintain network connectivity in isolated networks. The proposed protocol consists of three main components: neighborhood detection, broadcast suppression, and store-carryforward mechanisms. Each component is used to monitor the neighborhood to estimate the local topology, to broadcast storm problems by hand in dense traffic systems, and to maintain connections in sparse networks. In addition, they used the Region of Interest (ROI) to resolve two opposite directions on the highway when the sender node passed the data packet to the next node. Thus, the proposed network showed higher performance in terms of reliability, efficiency and scalability than the existing broadcasting protocol on the highway under multiple traffic conditions. However, the proposed protocol only considers the one-hop neighbor of VANET and the highway scenario. Viriyasitavat et. al proposed a broadcast route protocol and could be called UV-CAST (Urban Vehicle BroadCAST) for urban VANET[11]. The proposed protocol is designed to support all well-connected and well-connected network conditions in urban scenarios. Therefore, each vehicle can communicate with each other with zero infrastructure support. For this mechanism, the protocol uses a SCF mechanism to support isolated networks. In addition, the protocol can use regular personnel messages to avoid redundant messages generated by reruns. As a result, the proposed protocol displayed higher performance than other similar protocols in terms of reachability, reception distance, and network overhead in urban 
scenarios. Ros et. al proposed an adaptive broadcasting protocol called ABSM (Acknowledged Broadcast), a localized broadcasting protocol for VANETs [12]. ABSM working on the DSNES framework in an existing latency network. In this framework, the ABSM uses a connected dominant set (CDS) and a neighbor removal scheme (NES) based on 1-hop adjacent position information obtained from periodic beacons. Thus, the ABSM can improve protocol reliability and efficiency in the vehicle environment. However, the proposed ABSM could not apply the safety application because it does not take into account any delay restrictions on retransmission time limits.

\section{Mobility Prediction based Multi-Directional Broadcasting}

\subsection{Region of Interest (ROI)}

The appropriate ROI choices for distributing emergency messages depend on the various scenarios required, including highways and urban areas. The urgent message of ROI for urban scenarios should propagate in several directions, such as North, South, East and West, as there may be many intersections and changes in the direction of the vehicle at the intersection. Message distribution for highway scenarios may not work in the ROI of urban scenarios. And the storage-carry-forward (SCF) mechanism used in highway scenarios may not be an appropriate solution for urban areas. Because urban areas have many traffic lights and they often generate network connections. Thus, broadcasting protocols for urban scenarios can propagate messages in all (or many) directions of ROI and must incorporate more than one vehicle into the SCF task. Multiple SCF allocated vehicles may generate redundant broadcast messages. This solution ensures that redundant messages are not restricted only when the vehicle is assigned to receive signal messages from new vehicles.

\subsection{Adaptive Directional Sector}

The MPMB protocol adopts a directional sector method for adaptive propagation of emergency messages in several directions, depending on the type of road based on ROI. Directional sectors of a vehicle are defined as geographic groups of vehicles within the vehicle's transmission range. The maximum direction is limited to eight where the directional segment has a range of 45 degrees. If the direction of movement of the two vehicles is less than 45 degrees, it is considered to be moving in the same direction. The directional segment is selected from one direction to eight directions, depending on the road phase information obtained from the digital road map. In the MPMB protocol, the broadcast vehicle selects one vehicle with the largest LAT among adjacent vehicles as the next broadcast vehicle in each direction area.

\subsection{Mobility Prediction}

We consider the multi-directional broadcasting for both highway and urban scenarios through the mobility prediction of moving vehicles. The moving direction $(\theta)$ is calculated by the arctan function by using current location $(x, y)$ and future location $(x+\Delta \mathrm{t}, y+\Delta \mathrm{t})$. The speed $v i$ and direction $\theta i$ of vehicle $i$ during each time are distributed over the ranges of $(0, v \max )$ and $(0,2 \pi)$, respectively. When a beaconing time starts, the current position of vehicle $i$ is $(x i$, $y i$ ) and the current distance between vehicles $i$ and $j$ is $d i j$. After the beaconing time period $t$, the position of vehicle $i$ can be calculated using $\cos \theta t$ and $\sin \theta t$. And also, the predicted link available time (LAT) between vehicles $i$ and $j$ is obtained by relative speed and location in the range of them. 


\subsection{Algorithms}

We consider the multi-directional broadcasting for both highway and urban scenarios through direction sectors. In the MPMB, sender vehicles send beaconing message to its neighbors which belong to the transmission range of them. From it all sender vehicles can obtain neighbors which have the same moving direction with the sender vehicles.

The method of broadcasting process is used to deliver a message from the sender node to the destination. The broadcasting process of MPMB protocol basically follows the SCF scheme. Vehicles that need to transmit any data begin the broadcasting process based on neighbor nodes information built in the rebroadcasting vehicle selection step.

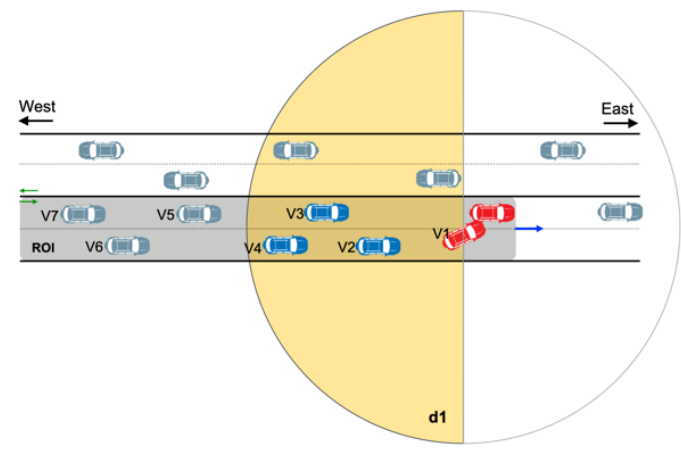

(a) Step1. V1 predicts the LAT of all vehicles within its d1.

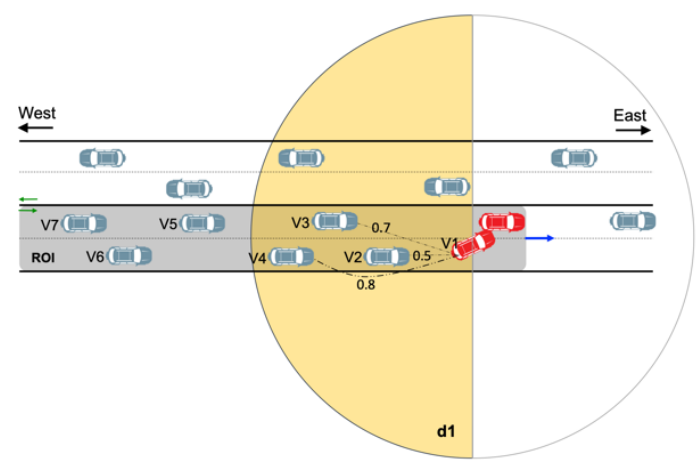

(c) Step3. V1 broadcasts the packet to all vehicles.

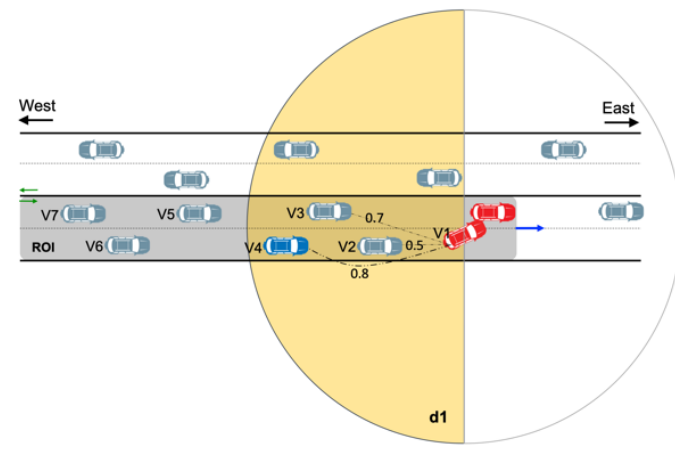

(b) Step2. V1 selects V4 as a rebroadcasting within its $\mathrm{d} 1$.

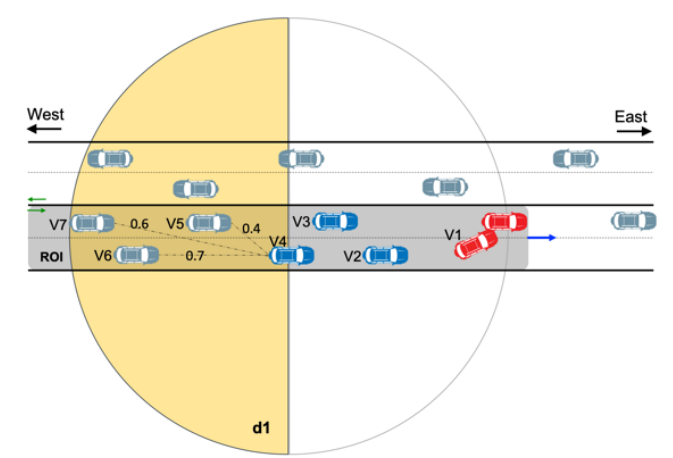

(d) Step4. V4 rebroadcasts the packet to its neighbor vehicles after selecting V6 as a rebroadcasting vehicle.

Figure 1. An highway example for MPMB protocol.

Fig. 1 and 2 illustrate how the MPMB protocol applies in highway and urban road environments. As shown in Fig. 1 (a), vehicle V1 calculates the LAT values of neighbor vehicles through periodic beacons in its rear $\mathrm{d} 1$ sector. If the vehicle $\mathrm{V} 1$ encounters an emergency message due to an accident such as a collision, select vehicle V4 with the highest value of the LAT values calculated before all neighboring vehicles in the d1 sector as the rebroadcasting vehicle and pass the broadcast message to the d1 sector (see Fig. 1 (b) and (c)). And then V4 also rebroadcasts the packet to its neighbor vehicles in the $\mathrm{d} 1$ sector after selecting 
V6 as a rebroadcasting vehicle. This process is repeated until the end of the Time-To-Live (TTL) of the original packet.

Fig. 2 shows the process of MPMB transmitting an emergency message in the context of a downtown intersection consisting of 4 sectors. Unlike highways, the situation on the urban roads selects the next broadcast vehicles with the largest LAT value for each 4 sectors and broadcasts the message.

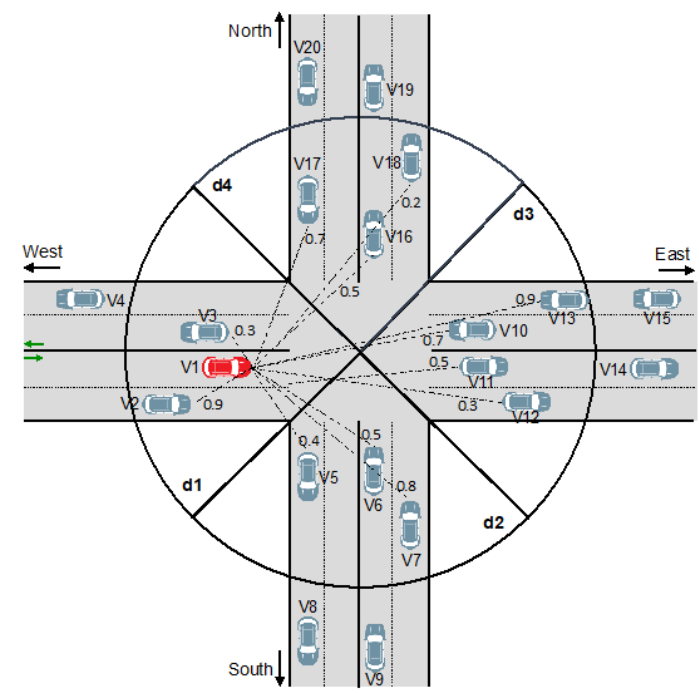

(a) Step1. V1 predicts the LAT of its all neighbor vehicles.

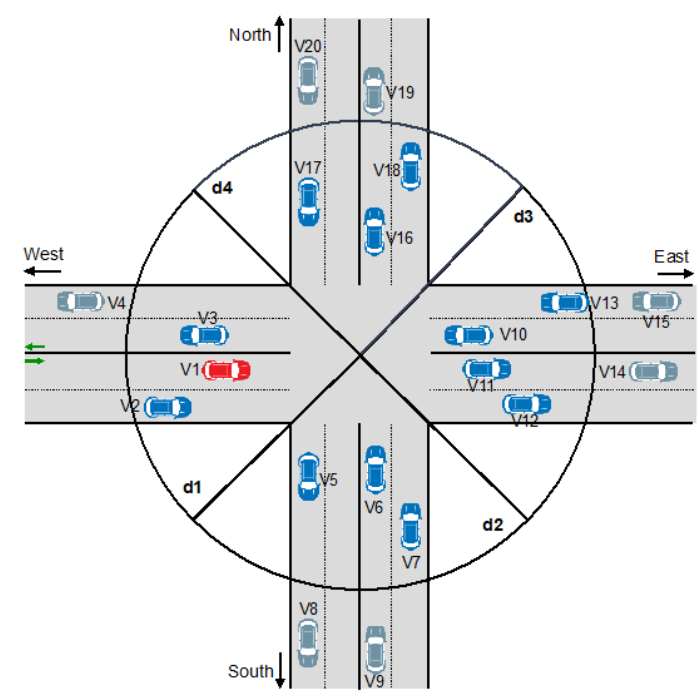

(c) Step3. V1 broadcasts the packet to its all neighbor vehicles.

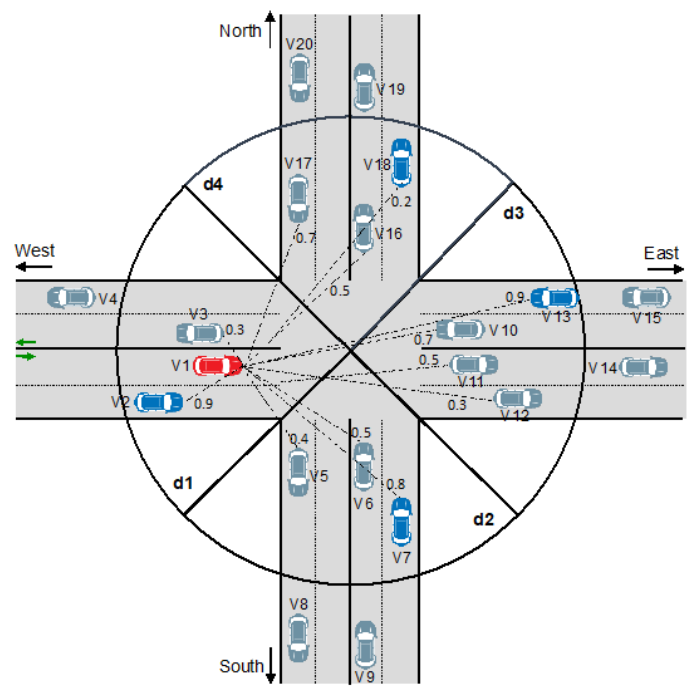

(b) Step2. V1 selects V2, V7, V13, and $\mathrm{V} 18$ as a rebroadcasting vehicle for $\mathrm{d} 1, \mathrm{~d} 2$, $\mathrm{d} 3$, and d4, respectively.

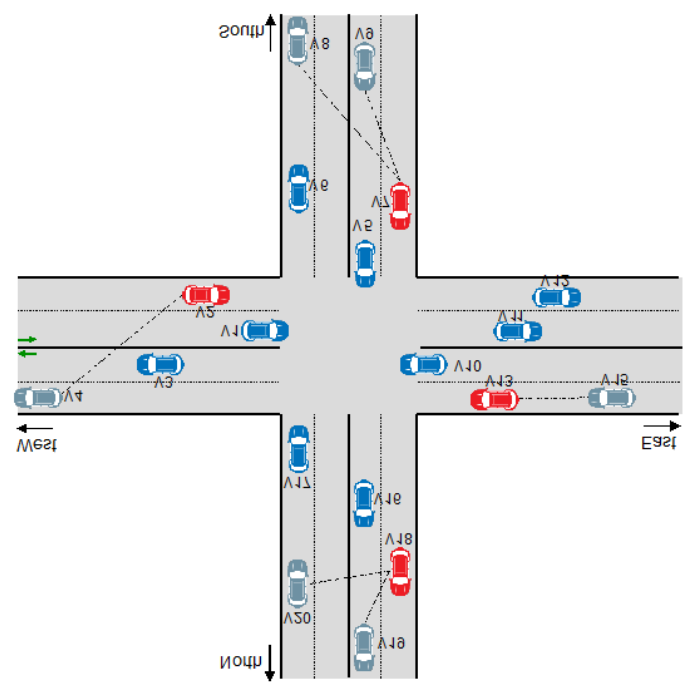

(d) Step4. V2, V7, V3, and V13 predicts recursively the LAT of their all neighbor vehicles.

Figure 2. An urban example for MPMB protocol.

\section{Conclusion}


In this paper, we described the development of a multi-directional broadcasting algorithm to propagate emergency messages to all vehicles not only on highways but also on urban roads. To this end, different sectors were applied to each region depending on the shape of the road. In addition, to select vehicles for retransmission in each sector, the mobility of each vehicle was predicted and the LAT values were obtained based on them. The vehicle with the highest LAT value becomes the retransmission vehicle for that sector. We are currently conducting final tests to predict mobility and to obtain LAT values.

\section{Acknowledgements}

Research Program through the National Research Foundation of Korea (NRF) funded by the Ministry of Education (NRF2016R1D1A1A09917662).

\section{References}

[1] V. Atanasovski, L. Gavrilovska. "Vehicular Sensor Networks: General Aspects and Implementation Issues". In: Gavrilovska L., Krco S., Milutinovic V., Stojmenovic I., Trobec R. (eds) Application and Multidisciplinary Aspects of Wireless Sensor Networks. Computer Communications and Networks, Springer, London (2011).

[2] W.Akyildiz, Y. S. Su, Cayirci E. Subramaniam. “A Survey on Sensor Networks”. IEEE Communications Magazine 40, pp.102-114 (2002).DOI: 10.1109/MCOM.2002.1024422

[3] M. J. Piran, G. R. Murthy, G. P. Babu. "Vehicular Ad Hoc and Sensor Networks; Principles and Challenges”. International Journal of Ad hoc, Sensor \& Ubiquitous Computing (IJASUC) 2, pp.38-49 (2011).

[4] W. Viriyasitavat, F. Bai, O. K. Tonguz. "UV-CAST: An Urban Vehicular Broadcast Protocol”. IEEE Communications Magazine, 49, pp.116-124 (2011).DOI: 10.1109/mcom.2011.6069718

[5] K. Abboud, W. Zhuang. "Modeling and Analysis for Emergency Messaging Delay in Vehicular Ad Hoc Networks”. In Proceedings of IEEE Global Telecommunications Conference, Honolulu, Hawaii, USA, 30 November - 4 December 2009; pp. 1-6 (2009). DOI: 10.1109/GLOCOM.2009.5425839

[6] Y. Zang, L. Stibor, H.-J. Reumerman. "Wireless Local Danger Warning using Inter-vehicle Communication in Highway Scenarios”. In Proceedings of the 14th IEEE European Wireless Conference, Prague, Czech Republic, 22-25 June 2008; pp.1-7 (2008). DOI: 10.1109/EW.2008.4623905

[7] S.-H. Cha. “A Survey of Broadcast Protocols for Vehicular Ad-hoc Net- works”. The Smart Computing Review (SmartCR) 4, pp.246-255 (2014).

[8] Y. Ohta, T. Ohta, E. Kohno, Y. Kakuda. “A Store-Carry-Forward-Based Data Transfer Scheme Using Positions and Moving Direction of Vehicles for VANETs”. In Proceedings of 10th International Symposium on Autonomous Decentralized Systems, Tokyo, Hiroshima, Japan, 23-27 March 2011, pp. 131-138 (2011). DOI: 10.1109/ISADS.2011.22

[9] G. Korkmaz, E. Ekici, F. Özgu ner U“ Özgu ner. "Urban multi-hop broad- cast protocol for inter-vehicle communication systems”. In Proceedings of the 1st ACM Vehicular Ad-hoc Networks Workshop, Philadelphia, PA, USA, 1 October 2004, pp.76-85 (2004). DOI: 10.1145/1023875.1023887

[10] O. K. Tonguz, N. Wisitponghan, F. Bai. "DV-CAST: a distributed vehicular broadcast protocol for vehicular ad hoc networks”, IEEE Wireless Communications, Vol.17, No.2, pp.47-57 (2010). DOI: 10.1109/MWC.2010.5450660

[11] W. Viriyasitavat, F. Bai, O. K. Tonguz. “UV-CAST: An urban vehicular broadcast protocol”. In Proceedings of the IEEE Vehicular Networking Conference (VNC), Jersey City, NJ, USA, 13-15 December 2010, pp. 25-32 (2010). DOI: 10.1109/MCOM.2011.6069718

[12] F. J. Ros, P. M. Ruiz, I. Stojmenovic. “Acknowledgment-based Broadcast Protocol for Reliable and Efficient Data Dissemination in Vehicular Ad- hoc Networks”. IEEE Transactions on Mobile Computing, 11, pp.33-46 (2012) DOI: 10.1109/tmc.2010.253 